\title{
Intraocular Pressure Responses to Systemic Autonomic Stimulation
}

\author{
LUMINA P. LANIGAN, CHARLES V. CLARK, DAVID W. HILL \\ London
}

\begin{abstract}
Summary
The intraocular pressure (IOP) responses to two standard tests of autonomic nerve function were studied in 15 healthy subjects, mean age 33 years (range: $20-64$ ). Both tests are based on cardiovascular reflexes: (1) The rise in diastolic blood pressure provoked by sustained isometric muscle contraction, a response mediated by the sympathetic nervous system and (2) The heart-rate responses to the Valsalva manoeuvre, a response mediated by the parasympathetic nervous system. During sustained isometric exercise, the mean IOP decreased significantly from baseline values of $12.60 \mathrm{mmHg}(\mathrm{SEM} 0.55)$ to $11.26 \mathrm{mmHg}(\mathrm{SEM} \mathrm{0.32}, \mathrm{p}<0.05)$ while mean diastolic blood pressure increased by $20.80 \mathrm{mmHg}$ (range: +12 to $+53 \mathrm{mmHg}$ ). However there was no direct correlation between them $(r=-0.05)$. Post exercise the mean IOP remained significantly lower than baseline values for five minutes $(p<0.01)$. During the Valsalva manoeuvre, there was a significant increase in IOP of 7.20 mmHg from a baseline of $12.80 \mathrm{mmHg}$ (SEM 0.45) to 20.00 $\mathrm{mmHg}$ (SEM 0.78, $\mathbf{p}<0.001$ ); during the recovery period, mean IOP was significantly lower than baseline values at $12.13 \mathrm{mmHg}(\mathrm{SEM} 0.39, \mathrm{p}<0.05)$. The mean Valsalva ratio was normal at 1.68 (range: 1.2-2.6). The possible mechanisms involved in these responses are discussed.
\end{abstract}

The role of the autonomic nervous system in the regulation of intraocular pressure is well recognised..$^{1-h}$ Current medications prescribed in the treatment of glaucoma-guttae adrenaline, guanethedine, timolol and pilocarpineact primarily via autonomic effects in the anterior segment of the eye. More recently, raised intraocular pressure has been associated with autonomic dysfunction. ${ }^{7}$ A number of simple well established tests of autonomic nerve function based on cardiovascular reflexes allow accurate assessment of the autonomic nervous system. ${ }^{8-13}$ The aim of this study was to assess the intraocular pressure responses in normal subjects to systemic autonomic stimulation.
The integrity of the sympathetic nervous system may be assessed by diastolic blood pressure responses to sustained isometric muscle contraction. The forearm muscles are utilised for this purpose and the experimental model is sustained handgrip..$^{2}$ The cardiovascular response to the sustained handgrip is reflex in nature ${ }^{14}$ and is thought to be initiated by stimuli from the exercising muscle. ${ }^{15}$ The rise in blood pressure is mediated partly by an increase in cardiac output which is heart-rate dependent $^{16}$ and partly by peripheral vasoconstriction mediated through alpha-adrenergic receptors of the peripheral autonomic nervous system. ${ }^{17} \mathrm{~A}$ rise of $\leqslant 10 \mathrm{mmHg}$

Correspondence to: L. P. Lanigan, Moorfields Eye Hospital, City Road, London EC1V 2PD.

From: Research Department of Ophthalmology, Royal College of Surgeons of England and Moorfields Eye Hospital, London. 
diastolic blood pressure is an abnormal response, $\geqslant 16 \mathrm{mmHg}$ normal and borderline if $<16 \mathrm{mmHg}$ and $>10 \mathrm{mmHg} .{ }^{13}$

The Valsalva manoeuvre stimulates a generalised autonomic response, involving both parasympathetic and sympathetic nervous systems, producing specific, well-defined effects on heart-rate and blood pressure. Parasympathetic nerve function may be assessed by heart-rate responses to the Valsalva manoeuvre. ${ }^{13}$ There are four phases in the haemodynamic responses to the Valsalva manoeuvre. Phase 1 is the onset of expiratory straining when the heart rate slows; it speeds up in response to the decreased venous return and falling blood pressure (Phase 2). Acute release of intrathoracic pressure (Phase 3) causes an increasing tachycardia due to a further drop in cardiac output secondary to increased pulmonary venous capacitance. Phase 4 (period of recovery) follows with a reflex bradycardia as blood pressure increases secondary to increasing cardiac output into a peripheral vasoconstricted vasculature. The change in heart rate during the Valsalva manoeuvre was used as an index of response and expressed as the Valsalva ratio. This is the ratio of the longest $\mathrm{R}-\mathrm{R}$ interval after the Valsalva to the shortest R-R interval during the Valsalva. A ratio of $\leqslant 1.10$ is defined as abnor$\mathrm{mal} ; \geqslant 1.20$ as normal and borderline if between 1.20 and $1.10 .{ }^{13}$

\section{Patients and Methods}

Fifteen healthy untrained volunteers were studied following informed consent. The mean age of the group was 33 years (range:

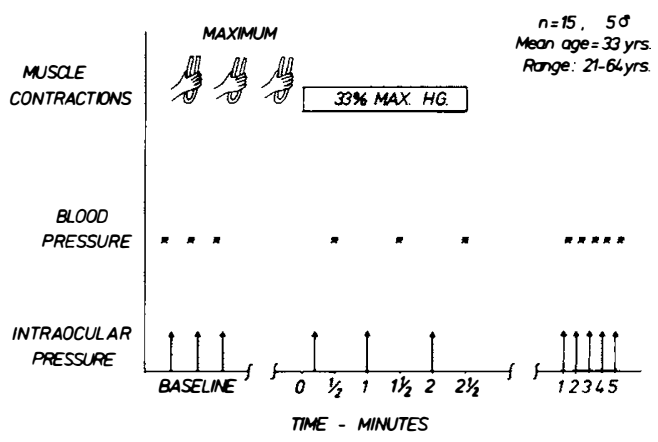

Fig. 1. Protocol for the handgrip study.
20--64 years) and five were male. The subject sat at the slit-lamp throughout the study.

\section{Diastolic blood pressure response to sustained isometric muscle contraction}

Each subject performed three sustained isometric muscle contractions using the dominant arm to establish their maximum voluntary contraction (MVC). Each maximum grip was sustained for less than two seconds. The handgrip dynamometer was devised by one of us (D.W.H.) and built in this Department. An inbuilt electronic audible alarm representing 33 per cent MVC was used for test contractions to alert the subject to weakening grip. Three baseline intraocular pressures (IOP) were recorded from one eye (whichever was preferred by the subject) using slit-lamp applanation tonometry after topical corneal anaesthesia with Benoxinate $(0.4$ per cent $)$ and sodium fluorescein $(0.25$ per cent). Three baseline blood pressures were recorded from the non-exercising arm using an automatic sphygmomanometer (COPAL-model UA-231). For the study 33 per cent MVC was held for 2-2.5 minutes, during which time three measurements of IOP and blood pressure were made at defined intervals as shown in Figure 1. On recovery five measurements of IOP and blood pressure were recorded at one minute intervals.

\section{Valsalva manoeuvre}

After a rest of 10-15 minutes, the subject was instructed to perform the Valsalva manoeuvre by blowing into a tube connected to a pressure transducer and to maintain an expiratory airway pressure of $35-40 \mathrm{mmHg}$ for 15 seconds.

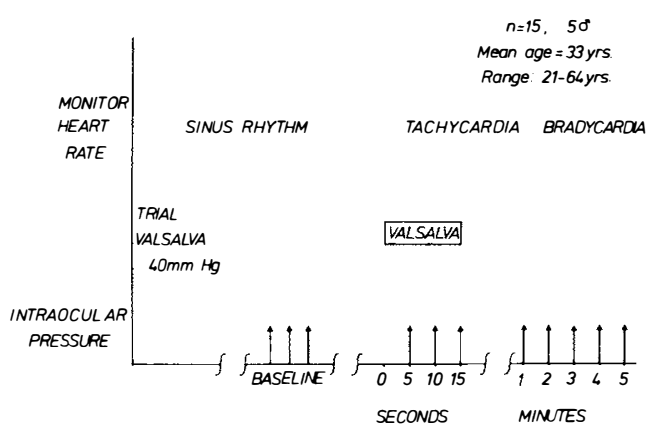

Fig. 2. Protocol for the Valsalva study. 
The heart rate was monitored throughout this study by a continuously recording electrocardiograph. All subjects were in sinus rhythm prior to commencing the Valsalva manoeuvre. Three baseline IOPs were recorded in the same eye and three more during the Valsalva manoeuvre as described in Figure 2. On recovery five measurements of IOP were made at one minute intervals.

\section{Statistical Analysis}

\section{Diastolic blood pressure response to sustained isometric muscle contraction}

Blood pressure and intraocular pressure at each specified period were recorded and the means and standard error of the means (SEM) calculated. Differences between peak change and mean baseline values and between mean recovery values and mean baseline values were then analysed for the group using the Wilcoxon paired signed rank test; statistical significance was defined as $\mathrm{p}<0.05$.

\section{Valsalva manoeuvre}

The means and the SEM of IOP and individual Valsalva ratios were calculated. Differences between peak change and mean baseline values and between mean recovery values and mean baseline values were analysed using the Wilcoxon paired signed rank test; statistical significance was defined as $\mathrm{p}<0.05$.

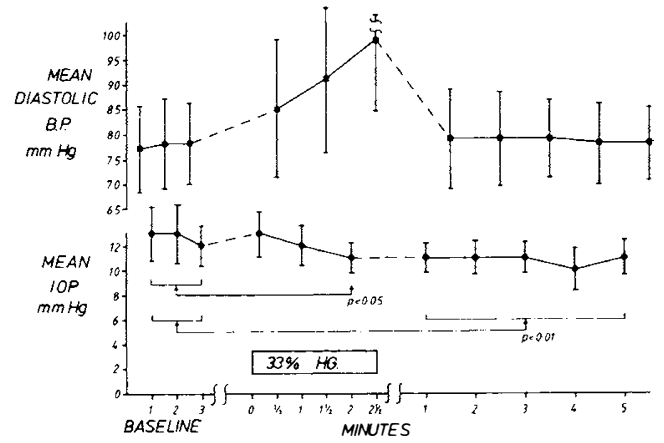

Fig. 3. Changes in intraocular pressure (IOP) and diastolic blood pressure (BP), during the handgrip (HG) study.

\section{Results}

1. Diastolic blood pressure response to sustained isometric muscle contraction

All subjects maintained the handgrip at 33 per cent MVC for two to 2.5 minutes. The mean rise in diastolic blood pressure during this static exercise was $20.80 \mathrm{mmHg}$ (range: +12 to $+53 \mathrm{mmHg}$ ), indicating a normal response (Fig. 3). Table I gives the individual changes in blood pressure and IOP in the 15 subjects. IOP (mean \pm SEM) decreased significantly from a baseline of $12.60 \pm 0.55 \mathrm{mmHg}$ to $11.26 \pm 0.32 \mathrm{mmHg}$ before handgrip was released $(\mathrm{p}<0.05)$. There was no direct correlation between the rise in diastolic blood pressure and the decrease in IOP $(r=-0.05$, $\mathrm{p}>0.05)$. Although diastolic blood pressure returned to near baseline levels within the first minute of recovery, IOP remained significantly lower than baseline values for at least five minutes $(\mathrm{p}<0.01)$, (Fig. 3).

\section{Valsalva manoeuvre}

There was a significant increase in mean IOP during the Valsalva manoeuvre of $7.20 \mathrm{mmHg}$ (56 per cent) from a baseline of $12.80 \pm 0.45$ $\mathrm{mmHg}$ to $20.00 \pm 0.78 \mathrm{mmHg}$ at 15 seconds $(\mathrm{p}<0.001)$ (Fig. 4). IOP remained lower than baseline values during the recovery period: mean recovery IOP was $12.13 \pm 0.39 \mathrm{mmHg}$ $(\mathrm{p}<0.05)$. The mean Valsalva ratio was normal at $1.68 \pm 0.11$ in all subjects. Table II gives the individual changes in IOP during this test with the individual Valsalva ratios.

\section{Discussion}

\section{Diastolic blood pressure response to} sustained isometric muscle contraction These results show that during generalised sympathetic nerve stimulation, effected by sustained isometric muscle contraction, there is a significant fall in IOP which remains below baseline levels for at least five minutes post exercise. No direct correlation however was found between the fall in IOP and the rise in diastolic blood pressure.

A complex study by Marcus et al. ${ }^{18}$ on the effects of sustained handgrip on IOP reported a trend similar to that in our study. They studied 6-7 subjects performing 50 per cent, 20 per cent maximal and 20 per cent submaxi- 
Table I Individual changes in diastolic blood pressure and intraocular pressure in response to sustained handgrip $(H G)$.

\begin{tabular}{ccccccc}
\hline $\begin{array}{c}\text { Subject } \\
\text { number }\end{array}$ & Age & $\begin{array}{c}\text { Baseline } \\
\text { diast. }{ }^{*} B P\end{array}$ & $\begin{array}{c}\text { BP rise } \\
\text { during } H G\end{array}$ & $\begin{array}{c}\text { Baseline } \\
\text { IOP* }\end{array}$ & $\begin{array}{c}\text { IOP change } \\
\text { during } H G\end{array}$ & $\begin{array}{c}\text { Recovery } \\
\text { IOP }\end{array}$ \\
\hline 1 & 61 & 69 & +21 & 13 & -1 & 9 \\
2 & 54 & 99 & +49 & 13 & -1 & 11 \\
3 & 20 & 68 & +22 & 13 & -2 & 10 \\
4 & 21 & 77 & +15 & 13 & -1 & 12 \\
5 & 20 & 68 & +22 & 8 & +2 & 8 \\
6 & 21 & 76 & +16 & 12 & -2 & 10 \\
7 & 21 & 81 & +14 & 16 & -4 & 12 \\
8 & 21 & 76 & +16 & 13 & -3 & 11 \\
9 & 21 & 72 & +23 & 13 & -3 & 10 \\
10 & 31 & 89 & +11 & 8 & +2 & 12 \\
11 & 23 & 75 & +20 & 13 & -1 & 11 \\
12 & 31 & 66 & +20 & 14 & -2 & 11 \\
13 & 22 & 76 & +25 & 12 & -2 & 11 \\
14 & 64 & 84 & +21 & 15 & -1 & \\
15 & 64 & 91 & +17 & 13 & -1 & \\
\hline
\end{tabular}

*Mean values, see text for details.

mal MVC. Close inspection of the data shown in their Figures 1-3, demonstrate that there was a small but definite rise in IOP soon after commencing the handgrip contraction, although this point was not discussed in their text. This initial rise although not statistically significant, was also seen in our study (Fig. 3) and may reflect the sudden increase in blood pressure at the beginning of the handgrip contraction. We have shown a post exercise decrease in IOP similar to that demonstrated by Marcus et al. and other authors. ${ }^{18-21}$ The exact mechanism for this is unclear; increase in serum osmolarity, a decrease in $\mathrm{pH}$ and an increase in serum lactate have all been put forward as possible explanations. Stewart et al. ${ }^{20}$ have shown that no significant alterations in the facility of aqueous outflow or in episcleral venous pressure occurs following exercise, thereby presumably excluding these factors as a possible influence. Marcus et al. demonstrated that the post exercise hypotension: (1) could be delayed by the application of a sphygmomanometer cuff on the exercising arm raised above systolic pressure ${ }^{18}$ and (2) was associated with increased blood lactate, decreased $\mathrm{pH}$ and increased serum osmolality. ${ }^{21}$ Both these observations indicate that some metabolic factor may be responsible for the post exercise ocular hypotension.

Unlike our experience Marcus failed to demonstrate any significant change in IOP during the exercise period. This may have been due to the differences in timing of IOP measurements in their study as compared to ours.

The graphic illustrations of the IOP changes in the study by Marcus et al. suggest that there was a direct correlation between the intensity of the handgrip contraction and the degree of post exercise ocular hypotension though with similar blood pressure responses. This would again support a local factor as a possible cause of the post exercise ocular hypotension. Moreover the absence of a direct association between blood pressure changes and post exercise decrease in IOP could be interpreted as contributory evidence to support this metabolite theory.

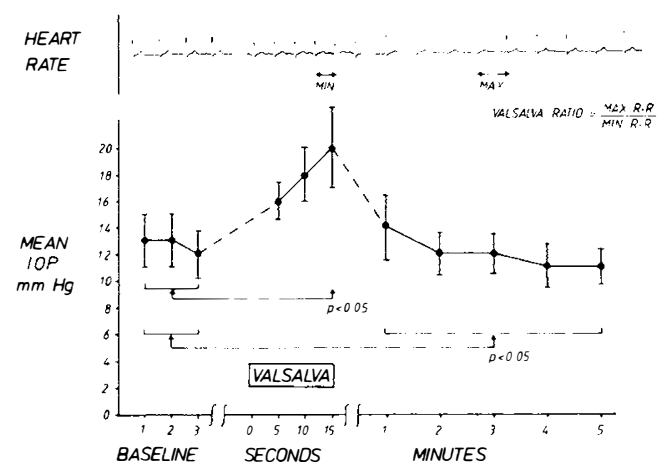

Fig. 4. Changes in intraocular pressure (IOP) and heart rate during the Valsalva study. 
Table II Individual changes in intraocular pressure during the Valsalva manoeuvre.

\begin{tabular}{|c|c|c|c|c|c|}
\hline $\begin{array}{l}\text { Subject } \\
\text { number }\end{array}$ & Age & $\begin{array}{l}\text { Valsalva } \\
\quad \text { ratio }\end{array}$ & $\begin{array}{c}\text { Baseline } \\
\text { IOP }^{*}\end{array}$ & $\begin{array}{c}\text { IOP rise } \\
\text { during } \\
\text { Valsalva }\end{array}$ & $\begin{array}{c}\text { Recovery } \\
\text { IOP* }\end{array}$ \\
\hline 1 & 61 & 1.2 & 13 & +4 & 11 \\
\hline 2 & 54 & 1.3 & 14 & +12 & 14 \\
\hline 3 & 20 & 1.8 & 13 & +7 & 12 \\
\hline 4 & 21 & 1.5 & 13 & +6 & 13 \\
\hline 5 & 20 & 1.8 & 9 & +11 & 10 \\
\hline 6 & 21 & 2.0 & 12 & +4 & 11 \\
\hline 7 & 21 & 2.0 & 14 & +8 & 13 \\
\hline 8 & 21 & 1.6 & 14 & +9 & 12 \\
\hline 9 & 21 & 1.6 & 13 & +7 & 11 \\
\hline 10 & 31 & 2.0 & 10 & +14 & 10 \\
\hline 11 & 23 & 2.6 & 13 & +2 & 13 \\
\hline 12 & 31 & 1.3 & 13 & $+\overline{5}$ & 11 \\
\hline 13 & 22 & 1.2 & 11 & +7 & 12 \\
\hline 14 & 64 & 1.2 & 16 & +6 & 15 \\
\hline 15 & 64 & 2.2 & 14 & +6 & 14 \\
\hline
\end{tabular}

*Mean values, see text for details.

Another possibility to be considered is the release of sympathomimetic amines during handgrip contraction. In our study, mean diastolic blood pressure increased immediately on starting the 33 per cent MVC from a baseline of $78 \mathrm{mmHg}$ to $99 \mathrm{mmHg}$ at $2-2.5$ minutes. Astrand et al. ${ }^{22}$ showed that heart rate and blood pressure increases were higher after static exercise than dynamic exercise. This cardiovascular response is largely attributable to an increase in sympathetic adrenergic vasomotor tone, resulting in constriction of resistance vessels..$^{15.22}$ Thus even moderately strong isometric muscle contractions have been found to result in a marked increase in arterial blood pressure. ${ }^{16}$ Hence one may postulate that because of the increased sympathetic tone, plasma adrenaline levels will be elevated and this may be effective in lowering IOP during handgrip. It may also explain the post exercise ocular hypotension observed both in our study and other studies, in view of the time required for the breakdown and re-uptake of circulating adrenaline. Exercise is known to increase plasma adrenaline levels significantly. ${ }^{23}$

\section{Valsalva manoeuvre}

The Valsalva manoeuvre is accompanied by a variety of complex haemodynamic events and involves both sympathetic and parasympathetic autonomic responses. The most likely mechanism for the significant rise in IOP during this manoeuvre is secondary to the effect of increased intrathoracic pressure on the great veins of the head and neck. Raised venous pressure is transmitted to the ocular veins and indeed a relationship exists between venous pressure and intrathoracic pressure: IOP $=6.51+0.82$ venous pressure. ${ }^{24}$ This effect would probably override the effect of the falling blood pressure which occurs simultaneously.

By contrast to our results in which all subjects showed a rise in IOP, Rosen et al. ${ }^{25}$ observed two principal patterns of ocular pressure changes during the Valsalva manoeuvre which they described as (1) the positive pattern, where the primary change was an increase in IOP and (2) the negative pattern where the IOP fell during the Valsalva. It is however possible for a subject to maintain artificially an expiratory airway pressure of 40 $\mathrm{mmHg}$ by inadvertently occluding the mouthpiece or tube with the tongue, but this would clearly not represent an intrathoracic pressure of $40 \mathrm{mmHg}$ and by definition would not be a Valsalva manoeuvre. In this situation the IOP would fall after a brief initial rise, simulating the negative pattern described above. Monitoring the heart rate changes during the Valsalva manoeuvre would be an objective indicator of the.subject's perform- 
ance, but was unfortunately omitted in the study by Rosen et al. ${ }^{25}$

The low IOP observed after the Valsalva manoeuvre, which was maintained for at least five minutes, is difficult to explain. Episcleral venous pressure is significantly raised during the Valsalva manoeuvre, thereby decreasing the facility of outflow and precipitating elevation of IOP. Upon termination of the manoeuvre, acute decrease in intrathoracic pressure will inevitably be accompanied by decreased episcleral venous pressure, increased outflow of aqueous and therefore lowering of IOP. If the changes in IOP were determined exclusively by the systemic blood pressure, rapid homeostasis of IOP would be anticipated. In fact, stabilisation of IOP required a period in excess of five minutes, suggesting an additional contributory factor-perhaps humoral or neurogenic-in the observed sequence of events. During phase 2 of the Valsalva manoeuvre where intrathoracic pressure is raised, blood pressure falls secondary to a decreased venous return; immediately on cessation of the Valsalva manoeuvre (phase 3 ), there is a further drop in blood pressure with increasing tachycardia due to the increased pulmonary capacitance. The falling blood pressure in phases 2 and 3 stimulates baroreceptors in the carotid sinus and aortic arch, and afferent impulses pass via the glossopharyngeal and vagus nerves to the medulla. Subsequent activation of the efferent sympathetic nerves causes peripheral vasoconstriction and may be associated with increased circulating adrenaline levels, with potentially significant effects in the postValsalva ocular hypotension.

The present study has demonstrated a significant association between stimulation of systemic autonomic cardiovascular reflexes and IOP, although the exact role of the autonomic nervous system in these acute IOP changes is unclear in view of the complex haemodynamic changes that occur; some possible mechanisms have been proposed. A greater understanding may be obtained by studying the IOP responses to autonomic stimulation in patients with systemic autonomic dysfuncton. This work is in progress.

Grateful thanks are due to Mr. J. Houseman, Senior
Technician, Royal College of Surgeons of England and to the patients and staff of Moorfields Eye Hospital, Holborn, for their cheerful cooperation in the study. L. P. Lanigan holds a Smith \& Nephew Fellowship in Ophthalmology. We thank Dr. D. J. Ewing, University of Edinburgh, for advice relating to autonomic function assessment.

\section{References}

'Barany EH: Transient increase in outflow facility after superior cervical ganglionectomy in rabbits. Arch Ophthalmol 1962, 67: 303-11.

2Sears ME and Barany EH: Outflow resistance and adrenergic mechanisms. Arch Ophthalmol 1960, 64: $839-48$.

${ }^{3}$ Richardson KT: Parasympathetic physiology and pharmacology. Surv Ophthalmol 1970, 14: 46176.

${ }^{4}$ Nagataki S and Brubaker EF: Effects of pilocarpine on aqueous humour formation in human beings. Arch Ophthalmol 1982, 100: 818-21.

${ }^{5}$ Mapstone R: Mechanisms in ocular hypertension. $\mathrm{Br}$ J Ophthalmol 1979, 63: 325-30.

${ }^{6}$ Mapstone R: Autonomic effects on aqueous outflow. Res Clin Forums 1981, 3: 35-9.

${ }^{7}$ Clark CV and Mapstone R: Autonomic neuropathy in ocular hypertension. Lancet 1985 , ii, 185-7.

${ }^{8}$ Ewing DJ, Borsey DQ, Bellavere F, Clarke BF: Cardiac autonomic neuropathy in diabetes: comparison of measures of $\mathrm{R}-\mathrm{R}$ interval variation. Diab etologia 1981, 21: 18-24.

${ }^{9}$ Levin AB: A simple test of cardiac function based on the heart rate changes induced by the Valsalva manoeuvre. Am J Cardiol 1966, 18: 90-9.

${ }^{10}$ Ewing DJ, Campbell IW, Murray A, Neilson JMM, Clarke BF: Immediate heart rate responses to standing: a simple test for autonomic neuropathy in diabetes. Br Med J 1978, 1: 145-7.

${ }^{11}$ Bellavere F and Ewing DJ: Autonomic control of the immediate heart rate response to lying down. Clin Sci 1982, 62: 57-64.

${ }^{12}$ Ewing DJ, Irving JB, Kerr F, Wildsmith JAW, Clarke BF: Cardiovascular responses to sustained handgrip in normal subjects and in patients with diabetes mellitus-a test of autonomic nerve function. Clin Sci Mol Med 1974, 46: 295-306.

${ }^{13}$ Ewing DJ, Campbell IW, Burt AA, Clarke BF: Vascular reflexes in diabetic autonomic neuropathy. Lancet 1973, ii: 1354-6.

${ }^{14}$ Coote JH, Hilton SM, Perez Gonzalez JF. The reflex nature of the pressor response to muscular exercise. J Ph ysiol 1971, 215: 789-804.

${ }^{15}$ Donald KW, Lind AR, McNicholl GW, Humphreys PW, Taylor SH, Staunton HP: Cardiovascular responses to sustained (static) contractions. Circ Res 1967, 20 (suppl. 1): 15-30.

${ }^{16}$ Lind AR, Taylor SF, Humphreys PW, Kennelly BM, Donald KW: The circulatory effects of sustained voluntary muscle contraction. Clin Sci 1964, 27: 229-44.

${ }^{17}$ Freyschuss U: Cardiovascular adjustments to 
somatomotor activation. Acta Physiol Scand 1970; suppl. 342.

${ }^{18}$ Marcus DF, Edjhauser HF, Maksud MG, Wiley RL: Effects of a sustained muscular contraction on human intraocular pressure. Clin Sci Mol Med 1974, 47: 249-57.

${ }^{19}$ Lempert $\mathrm{P}$ and Cooper K: The effect of exercise on intraocular pressure. Am J Ophthalmol 1967, 63: $1673-6$.

${ }^{20}$ Stewart RH, LeBlanc R, Becker B: Effects of exercise on aqueous dynamics. Am J Ophthalmol 1970, 69: 245-8.

${ }^{21}$ Marcus DF, Krupin T, Podos SM, Becker B: The effect of exercise on intraocular pressure-1. human beings. Invest Ophthalmol 1970, 9: 74952.

${ }^{22}$ Astrand I, Guharay A, Wahren J: Circulatory responses to arm exercises with different arm positions. J Appl Physiol 1968, 25: 528-32.

${ }^{23}$ De Jours P: Respirations. In Fenn WO, Rahn H. (Eds). Handbook of Physiology, Section 3; Chapter 25, Washington DC. Am Physiol Soc 1964, 1: 631-48.

${ }^{24} \mathrm{Macri} \mathrm{FJ}$ : Acetazolamide and the venous pressure of the eye. Arch Ophthalmol 1960, 63: 953-65.

${ }^{25}$ Rosen DA and Johnston VC. Ocular pressure patterns in the Valsalva manoeuvre. Arch Ophthalmol 1959, 62: 810-16. 